\title{
Minimal Energy Tree with 4 Branched Vertices
}

https://doi.org/10.1515/chem-2019-0013

received July 19, 2018; accepted November 27, 2018.

Abstract: The energy of a graph is defined as the sum of absolute values of the eigenvalues of its adjacency matrix. Let $\Omega(n, 4)$ be the class of all trees of order $n$ and having 4 branched vertices. In this paper, the minimal energy tree in the class $\Omega(n, 4)$ is found.

Keywords: Energy; Tree; Branch; Characteristic Polynomial; $r$ - matching.

\section{Introduction}

The neighboring atoms in a molecule play a significant role in studying the properties of chemical compounds, e.g. the boiling point of alkanes can be related to the branching pattern of their molecular skeleton. Therefore, based on the number of substituents attached to any carbon atom other than hydrogen in a chemical compound, carbon atoms are characterized as primary, secondary, tertiary or quaternary. The stability of the carbon compound depends much on the primary, secondary, tertiary or quaternary atoms. Moreover, the stability of a chemical compound is inversely related to the energy of the compound, i.e. the molecules with lesser energy are more stable. In a molecular graph of a carbon compound, the tertiary and quaternary atoms are refereed as branched vertices. Thus the analysis of minimal energy trees with certain branched vertices is worth studying. This leads to the motivation behind the study of minimal energy trees with certain branched vertices.

Let $G$ be a simple connected undirected graph of order $n$ having adjacency matrix $A(G)$. Let $\lambda_{r}, r=1,2,3, \ldots, n$ be the eigenvalues of the adjacency matrix of $G$. The energy of $G$ is denoted by $E(G)$ and is defined as

$$
E(G)=\sum_{r=1}^{n}\left|\lambda_{r}\right|
$$

\footnotetext{
*Corresponding author: Saira Hameed, Uzma Ahmad, University of the Punjab, Lahore, Pakistan , E-mails: saira-hameed@hotmail.com, uzma.math@pu.edu.pk
}

The characteristic polynomial of $\mathrm{G}$, denoted by $\Psi(G, x)$, is the same as the characteristic polynomial of $A(G)$ and is defined as

$$
\Psi(G, x)=\sum_{r=0}^{n} a_{r} x^{r}
$$

If a graph $G$ is bipartite, then $\Psi(G, x)$ is of the form

$$
\Psi(G, x)=\sum_{r=0}^{\left\lfloor\frac{n}{2} \mid\right.}(-1)^{r} m(G, r) x^{n-2 r},
$$

where $m(G, r)=1,2, \ldots,\left\lfloor\frac{n}{2}\right\rfloor$ are the numbers of $r$ matchings of G. Using Coulson's integral formula [1], the energy of a bipartite graph $\mathrm{G}$ of order $\mathrm{n}$ is defined as

$$
E(G)=\frac{2}{\pi} \int_{0}^{+\infty} \frac{1}{x^{2}} \operatorname{In}\left(\sum_{r=0}^{\left\lfloor\frac{n}{2}\right\rfloor} m(G, r) x^{2 r}\right) \mathrm{dx} .
$$

The energy is a graph-spectral-invariant which is a focus of great attention nowadays. It has a chemical motivation and is concerned with the total $\pi$-electron energy of graphs which represent a carbon-atom skeleton of conjugated hydrocarbons [2]. From a mathematical point of view, it was first introduced by Gutman in 1978 [3] and he proved that in the class of all trees on $n$ vertices, the star tree has the smallest energy and the path tree has the largest energy [2].

After that, the tree with the minimal energy having a diameter of at least $d$ for a given positive integer $d$ [5], the tree with minimal energy among the trees with $k$ pendent vertices [6] , the tree with the minimal energy in $\mathrm{T}(n, p)$ where $\mathrm{T}(n, p)$ is the set of trees of order $n$ with at most $p$ pendent vertices [7], were characterized. Moreover, the trees with a given domination number [11] and the trees with a given parameter [15], were also characterized. In 1999 Zhang and Li [21] studied the class of trees with $n$ vertices and perfect matching and proved that the minimal energy tree is a tree which is obtained by joining a pendent vertex to each vertex of $K_{\left(1, \frac{n}{2}-1\right)}$. Let $\Omega(n, m)$ be the class of all trees with $n$ vertices and exactly $m$ branched vertices.

In 2008 [8], 2010 [9] and 2012 [14], the authors determined up to the twelfth smallest energy trees of order $n$ which belong to either $\Omega(n, 1)$ or $\Omega(n, 2)$. Moreover, the 
trees with larger energies were also characterized in [12], [16], [17] and [18].

In 2015, MArín, Monsalve and Rada [19] showed that the trees $\mathrm{T}(2, n-4)$, given in Figure 1 and $\mathrm{T}(2,1, n$ 6 ), given in Figure 2 have minimal energy in the classes $\Omega(n, 2)$ and $\Omega(n, 3)$, respectively. They also found the trees with maximal energies in these classes which are given in Figures 3 \& 4. Recently, in 2018, Zhu and Yang [20] characterized the first four minimum energy trees in the class of all trees of order $n \geq 27$ and three branched vertices, that is,

$\mathrm{T}(2,1, n-6) \prec \mathrm{T}(2, n-7,2) \prec \mathrm{T}(3,1, n-7) \prec \mathrm{T}(2,2, n-7)$.

The motivation is to find the minimal energy tree in the class $\Omega(n, 4)$. In this paper, it is found that $\mathrm{T}(2,2, \mathrm{n}-8: 0)$, given in Figure 5, is the minimal energy tree in the class $\Omega(n, 4)$.

One of the methods used for the characterization of trees with respect to their energies is the quasi-order method which is defined as follows:

Definition 1.1. Let $T_{1}$ and $T_{2}$ be two trees of order $n$ having characteristic polynomials

$$
\Psi\left(T_{1}, x\right)=\sum_{r=0}^{\left\lfloor\frac{n}{2}\right\rfloor}(-1)^{r} m\left(T_{1}, r\right) x^{n-2 r}
$$

and

$$
\Psi\left(T_{2}, x\right)=\sum_{r=0}^{\left\lfloor\frac{n}{2}\right\rfloor}(-1)^{r} m\left(T_{2}, r\right) x^{n-2 r} .
$$

1. If $m\left(T_{1}, r\right) \leq m\left(T_{2}, r\right), 1 \leq r \leq\left\lfloor\frac{n}{2}\right\rfloor$ then $T_{1} \leqslant T_{2}$.

2. If $m\left(T_{1}, r\right)<m\left(T_{2}, r\right)$ for some $r, T_{1}<T_{2}$.

3. If $m\left(T_{1}, r\right)=m\left(T_{2}, r\right)$, for all $r$, then $T_{1} \sim T_{2}$.

Now, using equation (1),

1. if $T_{1} \leqslant T_{2}$ then $E\left(T_{1}\right) \leq E\left(T_{2}\right)$

2. if $T_{1} \prec T_{2}$ then $E\left(T_{1}\right)<E\left(T_{2}\right)$.

Lemma 1.1. ([4], [13]) Let $A$ and $X$ be trees different from $P_{1}$. Then

1. $A X_{n, i} \prec A X_{n, 3}$ for all $2 \leq i \leq n-2$ and $i \neq 3$;

2. $A X_{n, 2} \prec A X_{n, n-1}$ for all $n \geq 2, n \neq 3$;

3. $X_{n, 2} \prec X_{n, 4} \prec \ldots \prec X_{n, 5} \prec X_{n, 3} \prec X_{n, 1}$.

where $X_{n, i}=P_{n}(i, x) X$, is obtained by identifying the $i$ th vertex of the path $P_{n}$ to a vertex $x$ of $X$ and $A X_{n, i}=A(a, n) X_{n, i}$, is obtained by identifying the $i$ th and $n$th vertex of the path $P_{n}$ with a vertex $x$ of $X$ and a vertex $a$ of $A$, respectively.

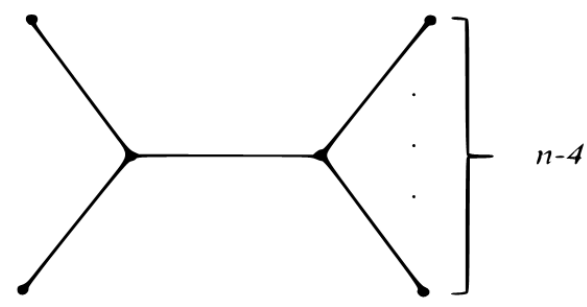

Figure 1: $\mathrm{T}(2, n-4)$.

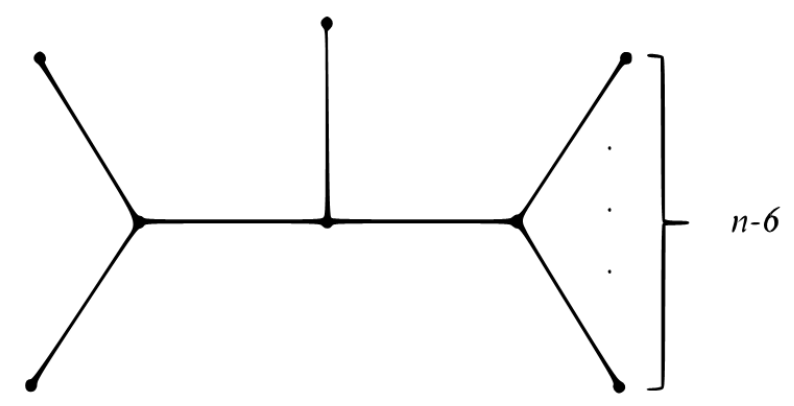

Figure 2: $\mathrm{T}(2,1, n-6)$.

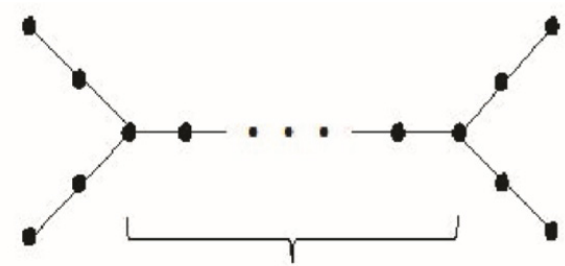

$n-8$

Figure 3: Maximal energy tree in $\Omega(n, 2)$.

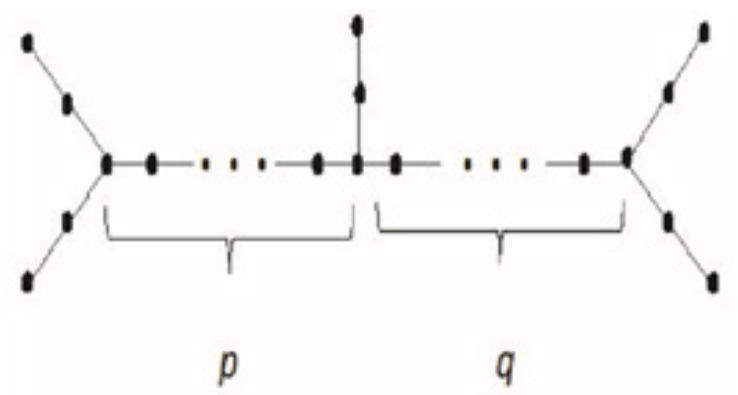

Figure 4: Maximal energy tree in $\Omega(n, 3)$.

Theorem 1.1. [19] $\mathrm{X}_{n, 2}<\mathrm{X}_{n, 1}$ for all $n \geq 2$.

The class $\Omega(n, 4)$ contains two subclasses, namely $\Omega^{\prime}(n, 4)$ consisting of all trees $T^{\prime}$ of the form (2) given in Figure 6 and $\Omega^{\star}(n, 4)$ consisting of all trees $T^{*}$ of the form (3) given in Figure 8. In section 2 and 3, the mathematical computations are carried out to find the minimal energy tree with in the general family of trees with four branched vertices. In section 4, the obtained results are implemented on branched alkanes with 10 and 11 carbon atoms. 


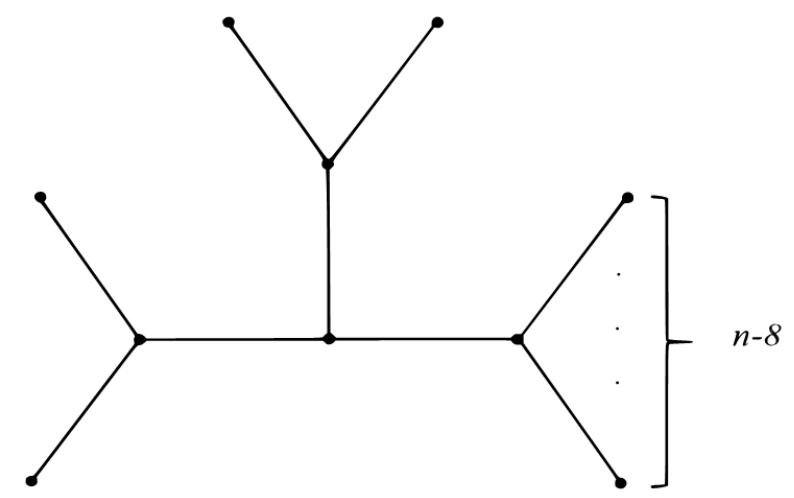

Figure 5: $\mathrm{T}(2,2, n-8: 0)$.

\section{Minimum Energy Tree in $\Omega^{\prime}(n, 4)$}

In this section, we will find the minimal energy tree in the class $\Omega^{\prime}(n, 4) \subset \Omega(n, 4)$ consisting of all trees $T^{\prime}$ of the form $T^{\prime}\left(\alpha_{1}, \alpha_{2}, \ldots, \alpha_{a} ; x ; \beta_{1}, \beta_{2}, \ldots, \beta_{b} ; y ; \gamma_{1}, \gamma_{2}, \ldots, \gamma_{c} ; z ; \delta_{1}, \delta_{2}, \ldots, \delta_{d}\right)$

given in Figure 6 , where $\alpha_{1}, \alpha_{2}, \ldots, \alpha_{\mathrm{a}}, \beta_{1}, \beta_{2}, \ldots, \beta_{\mathrm{b}}, \gamma_{1}, \gamma_{2}, \ldots$, $\gamma_{\mathrm{c}}, \delta_{1}, \delta_{2}, \ldots, \delta_{\mathrm{d}}$ are non-negative integers and $x, y, z$ are positive integers.

If $\alpha_{1}=\alpha_{2}=\cdots=\alpha_{\mathrm{a}}=\beta_{1}=\beta_{2}=\cdots=\beta_{\mathrm{b}}=\gamma_{1}=\gamma_{2}=\cdots=\gamma_{\mathrm{c}}=\delta_{1}=\delta_{2}=$ $\cdots=\delta_{\mathrm{d}}=x=y=z=1$, then $T^{\prime}$ is of the form $T^{\prime}(a, b, c, d)$, given in Figure 7.

Let

$$
\begin{gathered}
A^{\prime}(n, 4)=\left\{T^{\prime}(a, b, c, d): d \geq a \geq 2, b \geq 1, c \geq 1, a+b+\right. \\
+c+d=n-4\} \subset \Omega^{\prime}(n, 4)
\end{gathered}
$$

and

$$
B^{\prime}(n, 4)=\Omega^{\prime}(n, 4) \backslash A^{\prime}(n, 4) .
$$

Then

$$
\Omega^{\prime}(n, 4)=A^{\prime}(n, 4) \cup B^{\prime}(n, 4) .
$$

Lemma 2.1. Let $T^{\prime}=T^{\prime}\left(\alpha_{1}, \alpha_{2}, \ldots, \alpha_{a} ; x ; \beta_{1}, \beta_{2}, \ldots, \beta_{b} ; y ; \gamma_{1}\right.$, $\left.\gamma_{2}, \ldots, \gamma_{c} ; z ; \delta_{1}, \delta_{2}, \ldots, \delta_{d}\right) \in B^{\prime}(n, 4)$, then there exists a tree $T_{1}^{\prime}=T^{\prime}\left(p_{1}, p_{2}, p_{3}, p_{4}\right) \in A^{\prime}(n, 4)$ such that $T_{1}^{\prime}<T^{\prime}$.

Proof. The proof follows by the successive application of Lemma 1.1 and Theorem 1.1.

Lemma 2.2. Let $T^{\prime} \in A^{\prime}(n, 4)$, then

$$
T_{2}^{\prime}=T^{\prime}\left(p_{1}-1, p_{2}, p_{3}, p_{4}+1\right) \prec T_{1}^{\prime}=T^{\prime}\left(p_{1}, p_{2}, p_{3}, p_{4}\right) .
$$

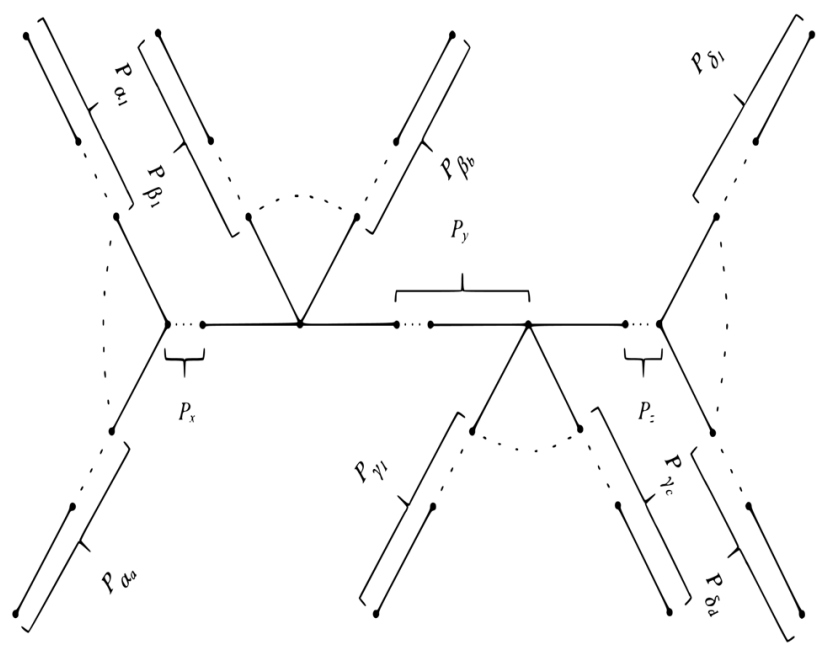

Figure 6: $T^{\prime}\left(\alpha_{1}, \alpha_{2}, \ldots, \alpha_{a} ; x ; \beta_{1}, \beta_{2}, \ldots, \beta_{b} ; y ; \gamma_{1}, \gamma_{2}, \ldots, \gamma_{c} ; z ; \delta_{1}, \delta_{2}, \ldots, \delta_{d}\right)$.

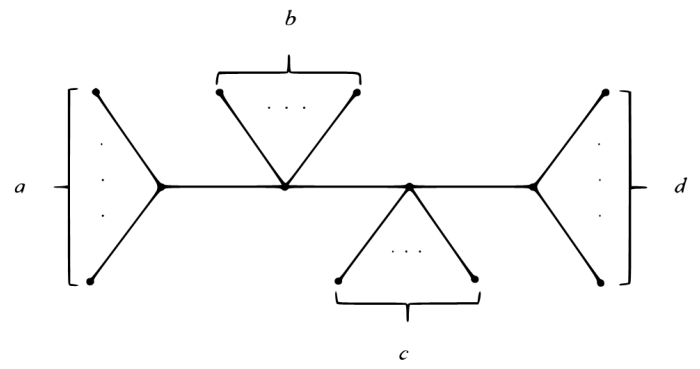

Figure 7: $T^{`}(a, b, c, d)$.

Proof. First we compute the characteristic polynomials of $T_{1}^{\prime}$ and $T_{2}^{\prime}$ as follows, $\Psi \quad\left(T_{1}^{\prime}\right)=x^{n}-(n-1) x^{n-2}+\left(p_{1} p_{2}+p_{1} p_{3}+p_{1} p_{4}+p_{2} p_{3}+p_{2} p_{4}+p_{3} p_{4}+2 p_{1}+p_{2}+p_{3}+\right.$
$\left.2 p_{4}+1\right) x^{n-4}-\left(p_{1} p_{2} p_{3}+p_{1} p_{2} p_{4}+p_{1} p_{3} p_{4}+p_{2} p_{3} p_{4}+p_{1} p_{4}+p_{1} p_{2}+p_{3} p_{4}\right) x^{n-6}+$
$\left(p_{1} p_{2} p_{3} p_{4}\right) x^{n-8}$

and

$\Psi\left(T_{2}^{\prime}\right)=x^{n}-(n-1) x^{n-2}+\left\{p_{1} p_{2}+p_{1} p_{3}+p_{1} p_{4}+p_{2} p_{3}+p_{2} p_{4}+p_{3} p_{4}+2 p_{1}+p_{2}+p_{3}+\right.$ $\left.2 p_{4}+1+\left(p_{1}-p_{4}-1\right)\right\} x^{n-4}-\left\{p_{1} p_{2} p_{3}+p_{1} p_{2} p_{4}+p_{1} p_{3} p_{4}+p_{2} p_{3} p_{4}+p_{1} p_{4}+p_{1} p_{2}+\right.$ $\left.p_{3} p_{4}+p_{2}\left(p_{1}-p_{4}-2\right)+p_{3}\left(p_{1}-p_{4}\right)+\left(p_{1}-p_{4}-1\right)\right\} x^{n-6}+\left\{p_{1} p_{2} p_{3} p_{4}+\right.$ $\left.p_{2} p_{3}\left(p_{1}-p_{4}-1\right)\right\} x^{n-8}$

We can easily see that $p_{1}-p_{4} \leq 0, p_{1}-p_{4}-1<0$ and $p_{1}-p_{4}-2$ $<0$. Therefore, $T_{2}^{\prime}<T_{1}^{\prime}$.

Lemma 2.3. Let $T_{3}^{\prime}=T^{\prime}\left(2, p_{2}, p_{3}, p_{4}\right) \in A^{\prime}(n, 4)$.

1. If $p_{4} \geq p_{2} \geq 2$, then $T_{4}^{\prime}=T^{\prime}\left(2, p_{2}-1, p_{3}, p_{4}+1\right)<T_{3}^{\prime}$.

2. If $p_{2}>p_{4} \geq 2$, then $T_{5}^{\prime}=T^{\prime}\left(2, p_{2}+1, p_{3}, p_{4}-1\right) \prec T_{3}^{\prime}$.

3. If $p_{4} \geq p_{3} \geq 2$, then $T_{6}^{\prime}=T^{\prime}\left(2, p_{2}, p_{3}-1, p_{4}+1\right) \prec T_{3}^{\prime}$.

4. If $p_{3}>p_{4} \geq 2$, then $T_{7}^{\prime}=T^{\prime}\left(2, p_{2}, p_{3}+1, p_{4}-1\right) \prec T_{3}^{\prime}$.

5. If $p_{2}>p_{3} \geq 2$, then $T_{8}^{\prime}=T^{\prime}\left(2, p_{2}+1, p_{3}-1, p_{4}\right) \prec T_{3}^{\prime}$.

6. If $p_{3} \geq p_{2} \geq 2$, then $T_{9}^{\prime}=T^{\prime}\left(2, p_{2}-1, p_{3}+1, p_{4}\right) \prec T_{3}^{\prime}$.

Proof. 1. The characteristic polynomials of $T_{3}{ }^{\prime}$ and $T_{4}{ }^{\prime}$ are, 
$\Psi\left(T_{3}^{\prime}\right)=x^{n}-(n-1) x^{n-2}+\left(3 p_{2}+3 p_{3}+4 p_{4}+p_{2} p_{3}+p_{2} p_{4}+p_{3} p_{4}+5\right) x^{n-4}-\left(2 p_{2}+2 p_{4}\right.$
$\left.+2 p_{2} p_{3}+2 p_{2} p_{4}+3 p_{3} p_{4}+p_{2} p_{3} p_{4}\right) x^{n-6}+\left(2 p_{2} p_{3} p_{4}\right) x^{n-8}$ and

$\Psi\left(T_{4}^{\prime}\right)=x^{n}-(n-1) x^{n-2}+\left\{3 p_{2}+3 p_{3}+4 p_{4}+p_{2} p_{3}+p_{2} p_{4}+p_{3} p_{4}+5+\left(p_{2}-p_{4}\right)\right\} x^{n-4}$ $-\left\{2 p_{2}+2 p_{4}+2 p_{2} p_{3}+2 p_{2} p_{4}+3 p_{3} p_{4}+p_{2} p_{3} p_{4}+p_{3}\left(p_{2}-p_{4}\right)\right.$
$\left.+2\left(p_{2}-p_{4}-1\right)\right\} x^{n-6}+\left\{2 p_{2} p_{3} p_{4}+2 p_{3}\left(p_{2}-p_{4}-1\right)\right\} x^{n-8}$

Since $p_{2}-p_{4} \leq 0$ and $p_{2}-p_{4}-1<0$, therefore $T_{4}^{\prime}<T_{3}^{\prime}$.

2. The characteristic polynomial of $T_{5}{ }^{\prime}$ is

$\Psi\left(T_{5}^{\prime}\right)=x^{n}-(n-1) x^{n-2}+\left\{3 p_{2}+3 p_{3}+4 p_{4}+p_{2} p_{3}+p_{2} p_{4}+p_{3} p_{4}+5+\left(p_{4}-p_{2}-\right.\right.$ 2) $\} x^{n-4}-\left\{2 p_{2}+2 p_{4}+2 p_{2} p_{3}+2 p_{2} p_{4}+3 p_{3} p_{4}+p_{2} p_{3} p_{4}+p_{3}\left(p_{4}-p_{2}-2\right)+\right.$ $\left.2\left(p_{4}-p_{2}-1\right)\right\} x^{n-6}+\left\{2 p_{2} p_{3} p_{4}+2 p_{3}\left(p_{4}-p_{2}-1\right)\right\} x^{n-8}$.

Since $p_{4}-p_{2}-1<0$ and $p_{4}-p_{2}-2<0$, therefore $T_{5}{ }^{\prime}<T_{3}{ }^{\prime}$.

3. The characteristic polynomial of $T_{6}{ }^{\prime}$ is

$\Psi\left(T_{6}^{\prime}\right)=x^{n}-(n-1) x^{n-2}+\left\{3 p_{2}+3 p_{3}+4 p_{4}+p_{2} p_{3}+p_{2} p_{4}+p_{3} p_{4}+5+\left(p_{3}-\right.\right.$ $\left.\left.p_{4}\right)\right\} x^{n-4}-\left\{2 p_{2}+2 p_{4}+2 p_{2} p_{3}+2 p_{2} p_{4}+3 p_{3} p_{4}+p_{2} p_{3} p_{4}+p_{2}\left(p_{3}-p_{4}-1\right)+\right.$ $\left.3\left(p_{3}-p_{4}\right)-1\right\} x^{n-6}+\left\{2 p_{2} p_{3} p_{4}+2 p_{2}\left(p_{3}-p_{4}-1\right)\right\} x^{n-8}$.

Since $p_{3}-p_{4} \leq 0$ and $p_{3}-p_{4}-1<0$, therefore $T_{6}^{\prime}<T_{3}{ }^{\prime}$.

4. The characteristic polynomial of $T_{7}{ }^{\prime}$ is

$\begin{aligned} \Psi\left(T_{7}^{\prime}\right)= & x^{n}-(n-1) x^{n-2}+\left\{3 p_{2}+3 p_{3}+4 p_{4}+p_{2} p_{3}+p_{2} p_{4}+p_{3} p_{4}+5+\left(p_{4}-p_{3}-\right.\right. \\ & 2)\} x^{n-4}-\left\{2 p_{2}+2 p_{4}+2 p_{2} p_{3}+2 p_{2} p_{4}+3 p_{3} p_{4}+p_{2} p_{3} p_{4}+p_{2}\left(p_{4}-p_{3}-1\right)+\right. \\ & \left.3\left(p_{4}-p_{3}\right)-5\right\} x^{n-6}+\left\{2 p_{2} p_{3} p_{4}+2 p_{2}\left(p_{4}-p_{3}-1\right)\right\} x^{n-8} .\end{aligned}$

Since $p_{4}-p_{3} \leq 0$ and $p_{4}-p_{3}-1<0$, therefore $T_{7}^{\prime} \prec T_{3}^{\prime}$.

5. The characteristic polynomial of $T_{8}^{\prime}$ is

$\Psi\left(T_{8}^{\prime}\right)=x^{n}-(n-1) x^{n-2}+\left\{3 p_{2}+3 p_{3}+4 p_{4}+p_{2} p_{3}+p_{2} p_{4}+p_{3} p_{4}+5+\left(p_{3}-p_{2}-\right.\right.$ 1) $\} x^{n-4}-\left\{2 p_{2}+2 p_{4}+2 p_{2} p_{3}+2 p_{2} p_{4}+3 p_{3} p_{4}+p_{2} p_{3} p_{4}+p_{4}\left(p_{3}-p_{2}-2\right)+\right.$ $\left.2\left(p_{3}-p_{2}\right)\right\} x^{n-6}+\left\{2 p_{2} p_{3} p_{4}+2 p_{4}\left(p_{3}-p_{2}-1\right)\right\} x^{n-8}$.

Since $\left(p_{3}-p_{2}-2\right)<0,\left(p_{3}-p_{2}-1\right)<0$ and $\left(p_{3}-p_{2}\right) \leq 0$, therefore, $T_{8}^{\prime}<T_{3}^{\prime}$.

6. The characteristic polynomial of $T_{9}^{\prime}$ is

$\Psi\left(T_{9}^{\prime}\right)=x^{n}-(n-1) x^{n-2}+\left\{3 p_{2}+3 p_{3}+4 p_{4}+p_{2} p_{3}+p_{2} p_{4}+p_{3} p_{4}+5+\right.$ $\left.\left(p_{2}-p_{3}-1\right)\right\} x^{n-4}-\left\{2 p_{2}+2 p_{4}+2 p_{2} p_{3}+2 p_{2} p_{4}+3 p_{3} p_{4}+p_{2} p_{3} p_{4}+\right.$ $\left.p_{4}\left(p_{2}-p_{3}\right)+2\left(p_{2}-p_{3}-2\right)\right\} x^{n-6}+\left\{2 p_{2} p_{3} p_{4}+2 p_{4}\left(p_{2}-p_{3}-1\right)\right\} x^{n-8}$

Since $\left(p_{2}-p_{3}-2\right)<0,\left(p_{2}-p_{3}-1\right)<0$ and $\left(p_{2}-p_{3}\right) \leq 0$ therefore, $T_{9}^{\prime} \prec T_{3}^{\prime}$.

Lemma 2.4. Let $^{\prime} T_{10}^{\prime}=T^{\prime}\left(2, p_{2}, p_{3}, 2\right) \in A^{\prime}(n, 4)$.

1. If $p_{3} \geq p_{2} \geq 2$, then $T_{11}^{\prime}=T^{\prime}\left(2, p_{2}-1, p_{3}+1,2\right)<T_{10}^{\prime}$.

2. If $p_{2} \geq p_{3} \geq 2$, then $T_{12}^{\prime}=T^{\prime}\left(2, p_{2}+1, p_{3}-1,2\right)<T_{10}^{\prime}$.

Proof. 1. The characteristic polynomials of $T_{10}{ }^{\prime}$ and $T_{11}{ }^{\prime}$ are

$\Psi\left(T_{10}^{\prime}\right)=x^{n}-(n-1) x^{n-2}+\left(5 p_{2}+5 p_{3}+p_{2} p_{3}+13\right) x^{n-4}-\left(6 p_{2}+6 p_{3}+4 p_{2} p_{3}+4\right) x^{n-6}$
$+\left(4 p_{2} p_{3}\right) x^{n-8}$ and

$\Psi\left(T_{11}^{\prime}\right)=x^{n}-(n-1) x^{n-2}+\left\{5 p_{2}+5 p_{3}+p_{2} p_{3}+13+\left(p_{2}-p_{3}-1\right)\right\} x^{n-4}-\left\{6 p_{2}+6 p_{3}\right.$ $\left.+4 p_{2} p_{3}+4+4\left(p_{2}-p_{3}-1\right)\right\} x^{n-6}+\left\{4 p_{2} p_{3}+4\left(p_{2}-p_{3}-1\right)\right\} x^{n-8}$.
Since $p_{2}-p_{3}-1<0$, therefore $T_{11}{ }^{\prime}<T_{10}{ }^{\prime}$.

3. The characteristic polynomial of $T_{12}{ }^{\prime}$ is

$\Psi\left(T_{12}^{\prime}\right)=x^{n}-(n-1) x^{n-2}+\left\{5 p_{2}+5 p_{3}+p_{2} p_{3}+13+\left(p_{3}-p_{2}-1\right)\right\} x^{n-4}-\left\{6 p_{2}+\right.$ $\left.6 p_{3}+4 p_{2} p_{3}+4+4\left(p_{3}-p_{2}-1\right)\right\} x^{n-6}+\left\{4 p_{2} p_{3}+4\left(p_{3}-p_{2}-1\right)\right\} x^{n-8}$.

Since $p_{3}-p_{2}-1<0$, therefore $T_{12}{ }^{\prime} \prec T_{10}{ }^{\prime}$.

Lemma 2.5. Let $T_{13}^{\prime}=T^{\prime}\left(2,1, p_{3}, p_{4}\right) \in A^{\prime}(n, 4)$.

1. If $p_{4} \geq p_{3} \geq 2$, then $T_{14}^{\prime}=T^{\prime}\left(2,1, p_{3}-1, p_{4}+1\right)<T_{13}^{\prime}$.

2. If $p_{3}>p_{4} \geq 2$, then $T_{15}^{\prime}=T^{\prime}\left(2,1, p_{3}+1, p_{4}-1\right) \prec T_{13}^{\prime}$.

Proof. 1. We compute the characteristic polynomials of $T_{13}{ }^{\prime}$ and $T_{14}{ }^{\prime}$ as follows,

$\Psi\left(T_{13}^{\prime}\right)=x^{n}-(n-1) x^{n-2}+\left(4 p_{3}+5 p_{4}+p_{3} p_{4}+8\right) x^{n-4}-\left(2+2 p_{3}+4 p_{4}+4 p_{3} p_{4}\right) x^{n-6}$ $+\left(2 p_{3} p_{4}\right) x^{n-8}$

and

$\Psi\left(T_{14}^{\prime}\right)=x^{n}-(n-1) x^{n-2}+\left\{4 p_{3}+5 p_{4}+p_{3} p_{4}+8+\left(p_{3}-p_{4}\right)\right\} x^{n-4}-\left\{2+2 p_{3}+4 p_{4}+\right.$ $\left.4 p_{3} p_{4}+4\left(p_{3}-p_{4}\right)-2\right\} x^{n-6}+\left\{2 p_{3} p_{4}+2\left(p_{3}-p_{4}-1\right)\right\} x^{n-8}$.

Since $p_{3}-p_{4} \leq 0$ and $p_{3}-p_{4}-1<0$, therefore, $T_{14}{ }^{\prime}<T_{13}{ }^{\prime}$.

1. The characteristic polynomial of $T_{15}{ }^{\prime}$ is

$\Psi\left(T_{15}^{\prime}\right)=x^{n}-(n-1) x^{n-2}+\left\{4 p_{3}+5 p_{4}+p_{3} p_{4}+8+\left(p_{4}-p_{3}-2\right)\right\} x^{n-4}$ $-\left\{2+2 p_{3}+4 p_{4}+4 p_{3} p_{4}+4\left(p_{4}-p_{3}-1\right)-2\right\} x^{n-6}+\left\{2 p_{3} p_{4}\right.$ $\left.+2\left(p_{4}-p_{3}-1\right)\right\} x^{n-8}$

Since $p_{4}-p_{3}-1<0$ and $p_{4}-p_{3}-2<0$, therefore, $T_{15}{ }^{\prime}<T_{13}{ }^{\prime}$.

Lemma 2.6. Let $T_{16}^{\prime}=T^{\prime}\left(2, p_{2}, 1, p_{4}\right) \in A^{\prime}(n, 4)$.

1. If $p_{4} \geq p_{2} \geq 2$, then $T_{17}^{\prime}=T^{\prime}\left(2, p_{2}-1,1, p_{4}+1\right)<T_{16}^{\prime}$.

2. If $p_{2}>p_{4} \geq 2$, then $T_{18}^{\prime}=T^{\prime}\left(2, p_{2}+1,1, p_{4}-1\right)<T_{16}^{\prime}$.

Proof. 1. The characteristic polynomials of $T_{16}{ }^{\prime}$ and $T_{17}{ }^{\prime}$ are

$\Psi\left(T_{16}^{\prime}\right)=x^{n}-(n-1) x^{n-2}+\left(4 p_{2}+5 p_{4}+p_{2} p_{4}+8\right) x^{n-4}-\left(4 p_{2}+5 p_{4}+3 p_{2} p_{4}\right) x^{n-6}$ $+\left(2 p_{2} p_{4}\right) x^{n-8}$

and

$\Psi\left(T_{17}^{\prime}\right)=x^{n}-(n-1) x^{n-2}+\left\{4 p_{2}+5 p_{4}+p_{2} p_{4}+8+\left(p_{2}-p_{4}\right)\right\} x^{n-4}$ $-\left\{4 p_{2}+5 p_{4}+3 p_{2} p_{4}+3\left(p_{2}-p_{4}\right)-2\right\} x^{n-6}+\left\{2 p_{2} p_{4}+2\left(p_{2}-p_{4}-1\right)\right\} x^{n-8}$

Since $p_{2}-p_{4}-1<0$ and $p_{2}-p_{4} \leq 0$, therefore, $T_{17}{ }^{\prime}<T_{16}{ }^{\prime}$.

2. The characteristic polynomial of $T_{18}{ }^{\prime}$ is

$\Psi\left(T_{18}^{\prime}\right)=x^{n}-(n-1) x^{n-2}+\left\{4 p_{2}+5 p_{4}+p_{2} p_{4}+8+\left(p_{4}-p_{2}-2\right)\right\} x^{n-4}$ $-\left\{4 p_{2}+5 p_{4}+3 p_{2} p_{4}+3\left(p_{4}-p_{2}-1\right)-1\right\} x^{n-6}+\left\{2 p_{2} p_{4}\right.$ $\left.+2\left(p_{4}-p_{2}-1\right)\right\} x^{n-8}$.

Since $p_{4}-p_{2}-1<0$ and $p_{4}-p_{2}-2<0$, therefore, $T_{18}{ }^{\prime}<T_{16}{ }^{\prime}$.

Lemma 2.7. Let $M^{\prime}=T^{\prime}(2,1,1, n-8)$ and $L^{\prime}=T^{\prime}(2,1, n$ -

9 ,2) belong to $A^{\prime}(n, 4)$, then $M^{\prime} \prec L^{\prime}$ for $n \geq 10$.

Proof. The characteristic polynomials of $L^{\prime}$ and $M^{\prime}$ are

$\Psi\left(L^{\prime}\right)=x^{n}-(n-1) x^{n-2}+(6 n-36) x^{n-4}-(10 n-80) x^{n-6}+(4 n-36) x^{n-8}$ $\Psi\left(M^{\prime}\right)=x^{n}-(n-1) x^{n-2}+(6 n-36) x^{n-4}-(8 n-60) x^{n-6}+(2 n-16) x^{n-8}$

For $n \geq 10$, 


$$
(10 n-80)-(8 n-60)=2 n-20 \geq 0
$$

and

$$
(4 n-36)-(2 n-16)=2 n-20 \geq 0 \text {. }
$$

So, $M^{\prime} \prec L^{\prime}$.

Theorem 2.1. Let $\mathrm{T}^{\prime} \in \Omega^{\prime}(n, 4), T^{\prime} \neq M^{\prime}$ and $n \geq 10$. Then $M^{\prime} \prec T^{\prime}$.

Proof. Since $\mathrm{T}^{\prime} \in \Omega^{\prime}(n, 4)$ and $T^{\prime} \neq M^{\prime}$, so by Lemma 2.1, there exists a tree

$$
T_{1}^{\prime}=T^{\prime}\left(p_{1}, p_{2}, p_{3}, p_{4}\right) \in \mathrm{A}^{\prime}(n, 4) \subset \Omega^{\prime}(n, 4),
$$

such that $T^{\prime} \succ T_{1}^{\prime}$. By Lemma 2.2, there also exists a tree

$$
T_{2}^{\prime}=T^{\prime}\left(2, p_{2}, p_{3}, p_{5}\right) \in \mathrm{A}^{\prime}(n, 4)
$$

such that $T_{1}^{\prime}>T_{2}{ }^{\prime}$.

1. If $p_{2} \leq p_{5}$, then by Lemma 2.3 (1), we can find a tree

$$
T_{3}^{\prime}=T^{\prime}\left(2,1, p_{3}, p_{6}\right) \in \mathrm{A}^{\prime}(n, 4)
$$

such that $T_{2}^{\prime}>T_{3}$.

(a) If $p_{3} \leq p_{6}$, then by Lemma $2.5, T_{3}{ }^{\prime}>M^{\prime}$

(b) If $p_{3}>p_{6}$, then by Lemma $2.5, T_{3}{ }^{\prime}>L^{\prime}$ and by Lemma 2.7 $L^{\prime}>M^{\prime}$.

2. If $p_{2}>p_{5}$, then by Lemma 2.3 (2), we can find a tree

$$
T_{4}^{\prime}=T^{\prime}\left(2, p_{7}, p_{3}, 2\right) \in \mathrm{A}^{\prime}(n, 4)
$$

such that $T_{2}{ }^{\prime}>T_{4}{ }^{\prime}$. Then either $p_{7} \geq p_{3}$ or $p_{3}>p_{\overrightarrow{7}}$ In both cases, by Lemma 2.4, we can find $L^{\prime}$ such that $T_{4}{ }^{\prime}>L^{\prime}$. Then using Lemma 2.7, $L^{\prime}>M^{\prime}$.

3. If $p_{3} \leq p_{5}$, then by Lemma 2.3 (3), we can find a tree

$$
T_{5}^{\prime}=T^{\prime}\left(2, p_{2}, 1, p_{8}\right) \in \mathrm{A}^{\prime}(n, 4)
$$

such that $T_{2}^{\prime}>T_{5}{ }^{\prime}$.

(a) If $p_{2} \leq p_{8}$, then by Lemma 2.6, $T_{5}{ }^{\prime}>M^{\prime}$.

(b) If $p_{2}>p_{8}$, then by Lemma $2.6, T_{5}{ }^{\prime}>L^{\prime}$ and by Lemma $2.7 L^{\prime}>M^{\prime}$.

4. If $p_{3}>p_{5}$, then by Lemma 2.3 (4), we can find a tree

$$
T_{6}^{\prime}=T^{\prime}\left(2, p_{2}, p_{9}, 2\right) \in \mathrm{A}^{\prime}(n, 4)
$$

such that $T_{2}{ }^{\prime}>T_{6}$. Then either $p_{9} \geq p_{2}$ or $p_{2}>p_{9}$. In both cases, by Lemma 2.4, we can find $L^{\prime}$ such that $T_{6}{ }^{\prime}>L^{\prime}$. Then using Lemma 2.7, $L^{\prime}>M^{\prime}$.

5. If $p_{2} \leq p_{3}$. Then by Lemma $2.3(6)$, we can find a tree

$$
T_{7}^{\prime}=T^{\prime}\left(2,1, p_{10}, p_{4}\right) \in \mathrm{A}^{\prime}(n, 4)
$$

such that $T_{2}{ }^{\prime}>T_{7}{ }^{\prime}$.

(a) If $p_{10} \leq p_{4}$, then by Lemma $2.5, T_{7}^{\prime}>M^{\prime}$

(b) If $p_{10}>p_{4}$, then by Lemma $2.5, T_{7}^{\prime}>L^{\prime}$ and by Lemma 2.7 $L^{\prime}>M^{\prime}$.

6. If $p_{2}>p_{3}$ then by Lemma 2.3 (5), we can find a tree

$$
T_{8}^{\prime}=T^{\prime}\left(2, p_{11}, 1, p_{4}\right) \in \mathrm{A}^{\prime}(n, 4)
$$

such that $T_{2}{ }^{\prime}>T_{8}{ }^{\prime}$.

(a) If $p_{11} \leq p_{4}$, then by Lemma $2.6, T_{8}{ }^{\prime}>M^{\prime}$.

(b) If $p_{11}>p_{4}$, then by Lemma 2.6, $T_{8}{ }^{\prime}>L^{\prime}$ and by Lemma $2.7 L^{\prime}>M^{\prime}$.

Hence $T^{\prime} \succ M^{\prime}$.

\section{Minimum Energy Tree in $\Omega^{*}(n, 4)$}

In this section, we will find the minimal energy tree in the class $\Omega^{\star}(n, 4)$ consisting of all trees $T^{\star}$ of the form

$$
T^{*}\left(\alpha_{1}, \alpha_{2}, \ldots, \alpha_{a} ; x ; \beta_{1}, \beta_{2}, \ldots, \beta_{b} ; y ; \delta_{1}, \delta_{2}, \ldots, \delta_{d} ; z ;, \gamma_{1}, \gamma_{2}, \ldots, \gamma_{c}\right),
$$

given in Figure 8, where $\alpha_{1}, \alpha_{2}, \ldots, \alpha_{a}, \beta_{1}, \beta_{2}, \ldots, \beta_{b}, \delta_{1}, \delta_{2}, \ldots, \delta_{d}, \gamma_{1}$, $\gamma_{2}, \ldots, \gamma_{c}$ are non-negative integers and $x, y, z$ are positive integers.

$$
\text { If } \alpha_{1}=\alpha_{2}=\ldots=\alpha_{\mathrm{a}}=\beta_{1}=\beta_{2}=\cdots=\delta_{1}=\delta_{2}=\cdots=\delta_{\mathrm{d}}=\gamma_{1}=\gamma_{2}=\cdots=
$$
$\gamma_{\mathrm{c}}=x=y=z=1$, then $T^{\star}$ is of the form $T^{\star}(a, b, d: c)$, given in Figure 9.

Let

$$
\begin{gathered}
A^{*}(n, 4)=\left\{T^{*}(a, b, d: c): d \geq b \geq a \geq 2, c \geq 0, a+b+d+\right. \\
+c=n-4\} \subset \Omega^{*}(n, 4)
\end{gathered}
$$

and

$$
B^{*}(n, 4)=\Omega^{*}(n, 4) \backslash A^{*}(n, 4) .
$$

Then

$\Omega^{*}(n, 4)=\mathrm{A}^{*}(n, 4) \cup \mathrm{B}^{*}(n, 4)$

Lemma3.1. Let $T^{*}=T^{*}\left(\alpha_{1}, \alpha_{2}, \ldots, \alpha_{a} ; x ; \beta_{1}, \beta_{2}, \ldots, \beta_{b} ; y ; \delta_{1}, \delta_{2}\right.$, $\left.\ldots, \delta_{d} ; z ;, \gamma_{1}, \gamma_{2}, \ldots, \gamma_{c}\right) \in B^{*}(n, 4)$ then there exists a tree $T_{1}^{*}=T^{*}\left(q_{1}, q_{2}, q_{3}: q_{4}\right) \in A^{*}(n, 4)$ such that $T_{1}^{*} \prec T^{*}$.

Proof. The proof follows by the successive application of Lemma 1.1 and Theorem 1.1.

Lemma 3.2. Let $T^{*} \in A^{*}(n, 4)$, then

$$
T_{2}^{*}=T^{*}\left(q_{1}-1, q_{2}, q_{3}+1: q_{4}\right) \prec T_{1}^{*}=T^{*}\left(q_{1}, q_{2}, q_{3}: q_{4}\right) .
$$




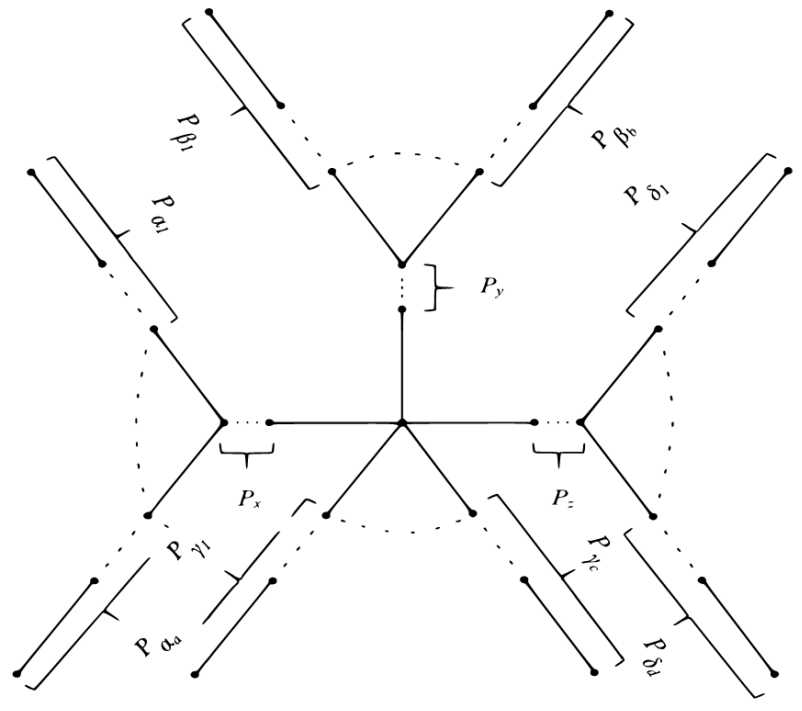

Figure 8: $T^{*}\left(\alpha_{1}, \alpha_{2}, \ldots, \alpha_{a} ; x ; \beta_{1}, \beta_{2}, \ldots, \beta_{b} ; y ; \delta_{1}, \delta_{2}, \ldots, \delta_{d} ; z ;, \gamma_{1}, \gamma_{2}, \ldots, \gamma_{c}\right)$.

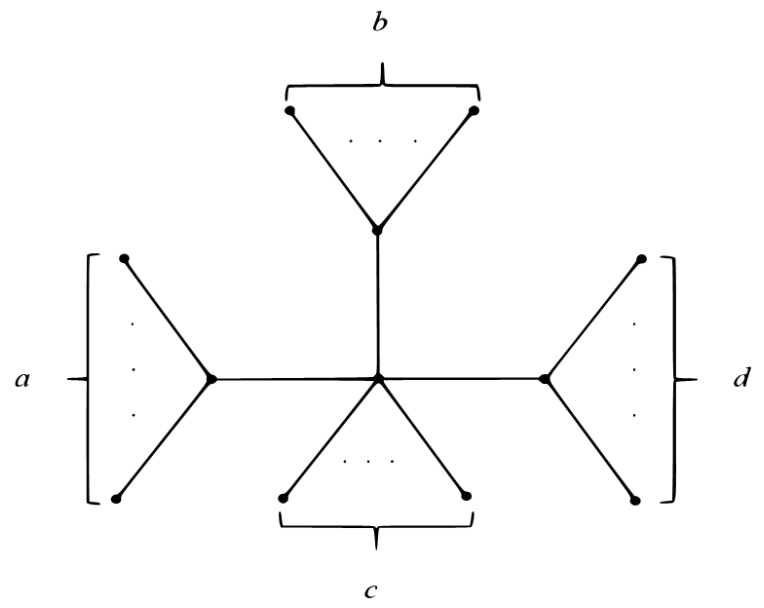

Figure 9: $T^{\star}(a, b, d: c)$.

Proof. First we compute the characteristic polynomials of $T_{1}^{*}$ and $T_{2}^{*}$ as follows,

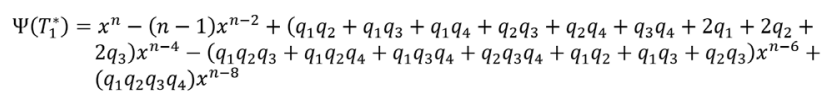

and

$\Psi\left(T_{2}^{*}\right)=x^{n}-(n-1) x^{n-2}+\left\{\left(q_{1} q_{2}+q_{1} q_{3}+q_{1} q_{4}+q_{2} q_{3}+q_{2} q_{4}+q_{3} q_{4}+2 q_{1}+2 q_{2}+\right.\right.$ $\left.\left.2 q_{3}\right)+\left(q_{1}-q_{3}-1\right)\right\} x^{n-4}-\left\{\left(q_{1} q_{2} q_{3}+q_{1} q_{2} q_{4}+q_{1} q_{3} q_{4}+q_{2} q_{3} q_{4}+q_{1} q_{2}+q_{1} q_{3}+\right.\right.$ $\left.\left.q_{2} q_{3}\right)+\left(q_{1}-q_{3}-1\right)\left(q_{2}+q_{4}+1\right)\right\} x^{n-6}+\left\{\left(q_{1} q_{2} q_{3} q_{4}\right)+q_{2} q_{4}\left(q_{1}-q_{3}-1\right)\right\} x^{n-8}$

Since $q_{1}-q_{3}-1<0$, therefore $T_{2}^{\star}<T_{1}^{\star}$.

Lemma 3.3. Let $T^{*} \in A^{*}(n, 4)$, then

$$
T_{4}^{*}=T^{*}\left(2, q_{2}-1, q_{3}+1: q_{4}\right) \prec T_{3}^{*}=T^{*}\left(2, q_{2}, q_{3}: q_{4}\right)
$$

Proof. First we compute the characteristic polynomials of $T_{3}{ }^{\star}$ and $T_{4}{ }^{\star}$ as follows,

$\begin{aligned} \Psi\left(T_{3}^{*}\right)=x^{n}- & (n-1) x^{n-2}+\left(4 q_{2}+4 q_{3}+2 q_{4}+q_{2} q_{3}+q_{2} q_{4}+q_{3} q_{4}+4\right) x^{n-4} \\ & -\left(q_{2} q_{3} q_{4}+3 q_{2} q_{3}+2 q_{2} q_{4}+2 q_{3} q_{4}+2 q_{2}+2 q_{3}\right) x^{n-6}+\left(2 q_{2} q_{3} q_{4}\right) x^{n-8}\end{aligned}$

and

$\Psi\left(T_{4}^{*}\right)=x^{n}-(n-1) x^{n-2}+\left\{\left(4 q_{2}+4 q_{3}+2 q_{4}+q_{2} q_{3}+q_{2} q_{4}+q_{3} q_{4}+4\right)+\left(q_{2}-q_{3}-\right.\right.$ 1) $\} x^{n-4}-\left\{\left(q_{2} q_{3} q_{4}+3 q_{2} q_{3}+2 q_{2} q_{4}+2 q_{3} q_{4}+2 q_{2}+2 q_{3}\right)+\left(q_{4}+3\right)\left(q_{2}-q_{3}\right.\right.$ 1) $\} x^{n-6}+\left\{\left(2 q_{2} q_{3} q_{4}\right)+2 q_{4}\left(q_{2}-q_{3}-1\right)\right\} x^{n-8}$.

Since $q_{2}-q_{3}-1<0$, therefore $T_{4}{ }^{\star}<T_{3}{ }^{\star}$.

Lemma 3.4. Let $T_{5}^{\star}=T^{\star}\left(2,2, q_{3}: q_{4}\right) \in A^{\star}(n, 4)$.

1. If $q_{3}>q_{4}$, then $T_{6}^{\star}=T^{\star}\left(2,2, q_{3}+1: q_{4}-1\right) \prec T_{5}{ }^{\star}$.

2. If $q_{4} \geq q_{3}$, then $T_{7}^{\star}=T^{\star}\left(2,2, q_{3}-1: q_{4}+1\right) \prec T_{5}^{\star}$.

Proof. 1. First we compute the characteristic polynomials of $T_{5}{ }^{\star}$ and $T_{6}{ }^{\star}$ as follows,

$$
\begin{gathered}
\Psi\left(T_{5}^{*}\right)=x^{n}-(n-1) x^{n-2}+\left(6 q_{3}+4 q_{4}+q_{3} q_{4}+12\right) x^{n-4} \\
-\left(4 q_{3} q_{4}+8 q_{3}+4 q_{4}+4\right) x^{n-6}+\left(4 q_{3} q_{4}\right) x^{n-8}
\end{gathered}
$$

and

$$
\begin{aligned}
& \Psi\left(T_{6}^{*}\right)=x^{n}-(n-1) x^{n-2}+\left\{\left(6 q_{3}+4 q_{4}+q_{3} q_{4}+12\right)+\left(q_{4}-q_{3}+1\right)\right\} x^{n-4} \\
& -\left\{\left(4 q_{3} q_{4}+8 q_{3}+4 q_{4}+4\right)+4\left(q_{4}-q_{3}\right)\right\} x^{n-6}+\left\{\left(4 q_{3} q_{4}\right)+4\left(q_{4}-q_{3}-1\right)\right\} x^{n-8}
\end{aligned}
$$

Since $q_{4}-q_{3}+1 \leq 0, q_{4}-q_{3}<0$ and $q_{4}-q_{3}-1<0$, therefore $T_{6}^{\star} \prec T_{5}^{\star}$.

2. The characteristic polynomial of $T_{7}^{\star}$ is

$$
\begin{aligned}
\Psi\left(T_{7}^{*}\right)= & x^{n}-(n-1) x^{n-2}+\left\{\left(6 q_{3}+4 q_{4}+q_{3} q_{4}+12\right)+\left(q_{3}-q_{4}-3\right)\right\} x^{n-4}-\left\{\left(4 q_{3} q_{4}+\right.\right. \\
& \left.\left.8 q_{3}+4 q_{4}+4\right)+4\left(q_{3}-q_{4}-2\right)\right\} x^{n-6}+\left\{\left(4 q_{3} q_{4}\right)+4\left(q_{3}-q_{4}-1\right)\right\} x^{n-8}
\end{aligned}
$$

Since $q_{3}-q_{4}-3<0, q_{3}-q_{4}-2<0$ and $q_{3}-q_{4}-1<0$, therefore $T_{7}^{\star}<T_{5}^{\star}$.

Lemma 3.5. Let $M^{*}=T^{*}(2,2, n-8: 0)$ and $T_{8}^{*}=T^{*}(2,2,2: n-10)$ belong to $A^{\star}(n, 4)$, then $M^{*} \prec T_{8}^{*}$ for $n>$ 10. In particular, $M^{*} \sim T_{8}^{*}$ for $n=10$.

Proof. The characteristic polynomials of $T_{8}^{\star}$ and $M^{\star}$ are

$$
\Psi\left(T_{8}^{*}\right)=x^{n}-(n-1) x^{n-2}+(6 n-36) x^{n-4}-(12 n-100) x^{n-6}+(8 n-80) x^{n-8}
$$

and

$$
\Psi\left(M^{*}\right)=x^{n}-(n-1) x^{n-2}+(6 n-36) x^{n-4}-(8 n-80) x^{n-6},
$$

respectively. Hence $M^{*} \prec T_{8}^{*}$.

Theorem 3.1. Let $T^{\star} \in \Omega^{\star}(n, 4), T^{\star} \neq M^{\star}$ and $n \geq 10$. Then $M^{*} \prec T^{*}$. 
Proof. Since $T^{\star} \in \Omega^{\star}(n, 4)$ and $T^{\star} \neq M^{\star}$, so by Lemma 3.1, there exists a tree

$$
T_{1}^{*}=T^{*}\left(q_{1}, q_{2}, q_{3}: q_{4}\right) \in \mathrm{A}^{*}(n, 4) \subset \Omega^{*}(n, 4)
$$

such that $T^{\star}>T_{1}^{\star}$.

By Lemmas 3.2 and 3.3, there exist trees

$$
T_{2}^{*}=T^{*}\left(2, q_{2}, q_{5}: q_{4}\right) \in \mathrm{A}^{*}(n, 4)
$$

and

$$
T_{3}^{*}=T^{*}\left(2,2, q_{6}: q_{4}\right) \in \mathrm{A}^{*}(n, 4)
$$

respectively, such that $T_{1}^{\star}>T_{2}{ }^{\star}>T_{3}$.

Now, if $q_{6}>q_{4}$, then by Lemma 3.4, $T_{3}^{\star}>M^{\star}$.

If $q_{6} \leq q_{4}$, then by Lemma 3.4, there exists a tree $T_{4}^{*}=T^{*}(2,2,2: n-10) \in \mathrm{A}^{*}(n, 4)$, such that $T_{3}{ }^{\star}>T_{4}{ }^{\star}$ and by Lemma 3.5. $T_{4}^{\star}>M^{\star}$.

Hence $M^{\star} \prec T^{\star}$.

Theorem 3.2. The minimal energy tree in $\Omega(n, 4)$ is $M^{*}=T^{*}(2,2, n-8: 0) \in \Omega^{\star}(n, 4)$.

Proof. The minimal energy tree in $\Omega(n, 4)$ is one of the minimal energy trees of its subclasses $\Omega^{\prime}(n, 4)$ and $\Omega^{\star}$ $(n, 4)$.

Since $M^{\prime}$ and $M^{\star}$ are the minimal energy trees in $\Omega^{\prime}(n, 4)$ and $\Omega^{\star}(n, 4)$, respectively, therefore $M^{\star} \prec M^{\prime}$ immediately follows from the comparison of the coefficients of $\Psi(M)$ and $\Psi\left(M^{\star}\right)$.

\section{Application in Structural Chemistry}

Alkanes are the simplest hydrocarbons but are very useful in the chemical industry. These compounds not only occur naturally in different products like natural gas and petroleum but are also used in the formation of different chemical products like lubricating oil, chloromethane, dichloromethane, trichloroethane (chloroform) and tetrachloromethane. Alkanes basically are of two kinds i.e., straight chain alkanes and branched alkanes. The stability of branched alkanes is better than that of straight chain alkanes. In other words, branched alkanes have less orbital energy as compared to straight ones.

But the question arises, "Among branched alkanes, which one is more stable?”

The proposed result partially answers this question. The branched alkanes contain tertiary or quaternary carbons. Thus, the molecular graphs of these alkanes are trees having branched vertices. According to the proposed
Table 1: The values of energies of different isomers of $\mathrm{C}_{11} \mathrm{H}_{24}$.

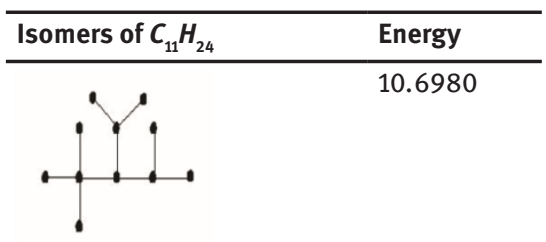

11.4548

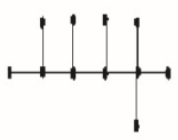

11.5756
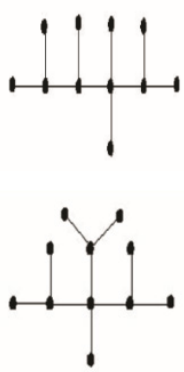

11.5994

11.8492
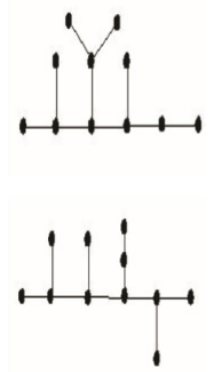

11.9086

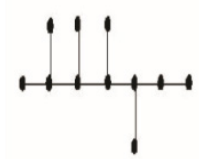

12.5266

result, the 3 - (2 - methyl ethyl $)$ - 2,2,4 - trimethylpentane and 3-(2-methylethyl)-2,4-diimethylpentane has minimal energy among all isomers of branched alkanes with 10 and 11 carbon atoms, respectively having exactly four branched carbon atoms and hence are more stable. To verify the result, we take all isomers of branched alkanes with 10 and 11 carbon atoms having exactly four branched carbon atoms. Then their energies are computed. The tables 1 \& 2 give all possible such branched alkanes with $n=10,11$ and their corresponding orbital energies. From Table 1, it can be observed that 3-(2-methylethyl) -2,2,4trimethylpentane has minimal energy among all branched 
Table 2: The values of energies of different isomers of $C_{10} H_{24}$.

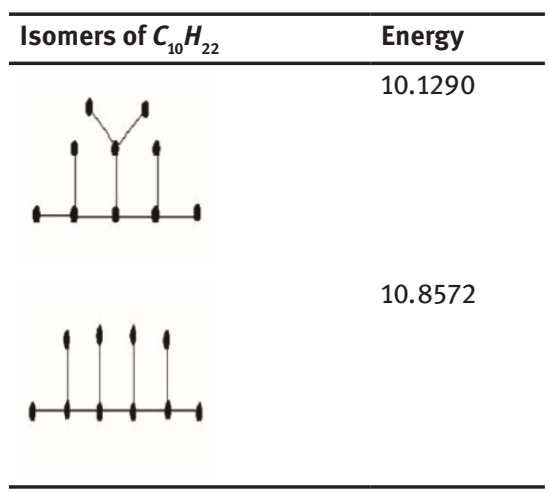

alkanes with 10 carbon atoms and having four branched carbon atoms. Similarly from Table 2, the alkane 3-(2methylethyl)-2,4-diimethylpentane has minimal energy among all branched alkanes with 11 carbon atoms and having four branched carbon atoms. In both cases, the minimal energy branched alkanes exactly match with the proposed result as given in Section 3.

Conclusion 4.1. The minimum energy tree in the class of all trees of order $n \geq 10$ with 4 branched vertices is $T(2,2, n-8: \quad 0)=T^{*}(2,2, n-8,0) \in \Omega(n, 4)$. As an application in structural chemistry, energies of 2 classes of alkanes with 10 and 11 carbon atoms respectively and having exactly 4 branched carbon atoms, are computed and it is observed that 3 - (2- methyl ethyl)-2,2,4-trimethylpentane has minimal energy among all branched alkanes with 10 carbon atoms and having four branched carbon atoms and 3-(2-methylethyl)- 2,4 - diimethylpentane has minimal energy among all branched alkanes with 11 carbon atoms and having four branched carbon atoms. In both cases, the minimal energy branched alkanes exactly match with the proposed result.

Ethical approval: The conducted research is not related to either human or animal use.

Conflict of interest: Authors declare no conflict of interest.
[4] Gutman I., Zhang F., On the ordering of graphs with respect to their matching numbers, Discr. Appl. Math., 1986, 15(1), 22-33.

[5] Yan W., Ye L., On the minimal energy of trees with a given diameter, Appl. Math. Lett., 2005, 18, 1046-1052.

[6] Yu, A. Lv X., Minimal energy of trees with $k$ pendent vertices, Lin. Algebra Appl., 2006, 418, 625-633.

[7] Ye L., Yuan X., On the minimal energy of trees with a given number of pendant vertices, MATCH Commun. Math. Comput. Chem.2007, 57, 193-201.

[8] Li N., Li S., On the extremal energy of trees, MATCH Commun. Math. Comput. Chem., 2008, 59, 291-314.

[9] Wang W., Kang L., Ordering of the trees by minimal energy, J. Math. Chem., 2010, 47(3), 937-958.

[10] Ou J., On ordering chemical trees by energy, MATCH Commun. Math. Comput. Chem., 2010, 64, 157-168.

[11] He C., Wu B., Yu Z., On the energy of trees with given domination number, MATCH Commun. Math. Comput. Chem., 2011, 61, 169-180.

[12] Huo B., Ji S., Li X., Shi Y., Complete solution to a conjecture on the fourth maximal energy tree, MATCH Commun. Math. Comput. Chem., 2011, 66, 903-912.

[13] Li X., Shi Y., Gutman I., Graph Energy, Springer, New York, (2012).

[14] Shan H. and Shao J., The proof of a conjecture on the comparison of the energies of trees, J. Math. Chem., 2012, 50(10), 2637-2647.

[15] Zhu J., Minimal energies of trees with given parameters, Lin. Algebra Appl., 2012, 436, 3120-3131.

[16] Andriantiana E. O. D., More trees with large energy, MATCH Commun. Math. Comput. Chem., 2012, 68 (2012), 675-695.

[17] Shan H., Shao J., Zhang L., He C., Proof of a conjecture on trees with Irage energy, MATCH Commun. Math. Comput. Chem., 2012, 68, 703-720.

[18] Gutman I., Furtula B., Andriantiana E. O. D., Cvetić M., More trees with large energy and small size, MATCH Commun. Math. Comput. Chem., 2012, 68, 697-702.

[19] MArín C., Monsalve J., Rada J., Maximum and minimum energy trees with two and three branched vertices, MATCH Commun. Math. Comput. Chem., 2015, 74, 285 - 306.

[20] Zhu J., Yang J., Minimal Energies of Trees with Three Branched Vertices, MATCH Commun. Math. Comput. Chem., 2018, 79, $263-274$.

[21] Zhang F., Li H., On acyclic conjugated molecules with minimal energies, Discrete. Applied. Mathematics, 1999, 92, 71-84.

\section{References}

[1] Coulson C. A., On the calculation of the energy in unsaturated hydrocarbon molecules, Proc. Cambridge Phil. Soc. 1940, 36(2), 201-203.

[2] Gutman I., Acyclic systems with extremal Hückel $\pi$-electron energy, Theor. Chim. Acta, 1977, 45, 79-87.

[3] Gutman I., The energy of a graph, Ber. Math. Statist. Sekt. Forschungsz. Graz, 1978, 103, 1-22. 